University of Wollongong

Research Online

Australian Institute for Innovative Materials -

Papers

Australian Institute for Innovative Materials

$1-1-2017$

\title{
Thermodynamics of quasi-2D electron gas at BFO/Si interface probed with THz time-domain spectroscopy
}

Xiankuan Liu

Shanghai University

Jiadong Zhang

Shanghai University

Zeyu Zhang

Shanghai University

Xian Lin

Shanghai University

Yang Yu

Shanghai University

See next page for additional authors

Follow this and additional works at: https://ro.uow.edu.au/aiimpapers

Part of the Engineering Commons, and the Physical Sciences and Mathematics Commons

Research Online is the open access institutional repository for the University of Wollongong. For further information contact the UOW Library: research-pubs@uow.edu.au 


\title{
Thermodynamics of quasi-2D electron gas at BFO/Si interface probed with $\mathrm{THz}$ time-domain spectroscopy
}

\author{
Abstract \\ An interface is constructed based on a bismuth ferrite oxide (BFO) thin film and p-type silicon, and the \\ temperature dependence of the interface properties has been studied systematically using terahertz time- \\ domain spectroscopy. The BFO/Si interface exhibits quasi-two-dimension electron gas (2DEG) transport \\ in the temperature range of 80 to $140 \mathrm{~K}$ : the electrons at the interface possess large electron mobility \\ $(\sim 106 \mathrm{~cm} 2 / \mathrm{V} \mathrm{s})$ and long scattering time ( $\sim 100 \mathrm{ps})$. As the temperature is higher than $140 \mathrm{~K}$, an abrupt \\ decrease in $\mathrm{THz}$ interface conductivity is observed due to the breakdown of the 2D EG induced by the \\ surface phase transition in the BFO thin film. Our result reveals that the interface formed between BFO \\ and Si provides a special platform for designing and fabricating $\mathrm{THz}$ photonic devices.

\section{Disciplines} \\ Engineering | Physical Sciences and Mathematics

\section{Publication Details} \\ Liu, X., Zhang, J., Zhang, Z., Lin, X., Yu, Y., Xing, X., Jin, Z., Cheng, Z. \& Ma, G. (2017). Thermodynamics of \\ quasi-2D electron gas at BFO/Si interface probed with $\mathrm{THz}$ time-domain spectroscopy. Applied Physics \\ Letters, 111 (15), 152906-1-152906-5.

\section{Authors} \\ Xiankuan Liu, Jiadong Zhang, Zeyu Zhang, Xian Lin, Yang Yu, Xiao Xing, Zuanming Jin, Zhenxiang Cheng, \\ and Guohong $\mathrm{Ma}$
}




\section{Thermodynamics of quasi-2D electron gas at BFO/Si interface probed with THz time- domain spectroscopy}

Xiankuan Liu, Jiadong Zhang, Zeyu Zhang, Xian Lin, Yang Yu, Xiao Xing, Zuanming Jin, Zhenxiang Cheng, and Guohong Ma

Citation: Appl. Phys. Lett. 111, 152906 (2017);

View online: https://doi.org/10.1063/1.4989667

View Table of Contents: http://aip.scitation.org/toc/apl/111/15

Published by the American Institute of Physics

\section{Articles you may be interested in}

Enhanced biexciton emission from single quantum dots encased in $\mathrm{N}$-type semiconductor nanoparticles

Applied Physics Letters 111, 153106 (2017); 10.1063/1.4989605

E-field controlled phase transformation in bismuth ferrite thin films, and effect of laser energy density Applied Physics Letters 111, 152905 (2017); 10.1063/1.4997017

Oxygen deficiency and cooling field driven vertical hysteretic shift in epitaxial $\mathrm{SrRuO} 3 / \mathrm{SrTiO}_{3}$ heterostructures Applied Physics Letters 111, 152405 (2017); 10.1063/1.5000866

Static negative capacitance of a ferroelectric nano-domain nucleus

Applied Physics Letters 111, 152902 (2017); 10.1063/1.4989391

Amorphousness induced significant room temperature ferromagnetism of $\mathrm{TiO}_{2}$ thin films

Applied Physics Letters 111, 152408 (2017); 10.1063/1.4999912

Spectroscopic studies of chiral perovskite nanocrystals

Applied Physics Letters 111, 151102 (2017); 10.1063/1.5001151

\section{Scilight}

Sharp, quick summaries illuminating the latest physics research

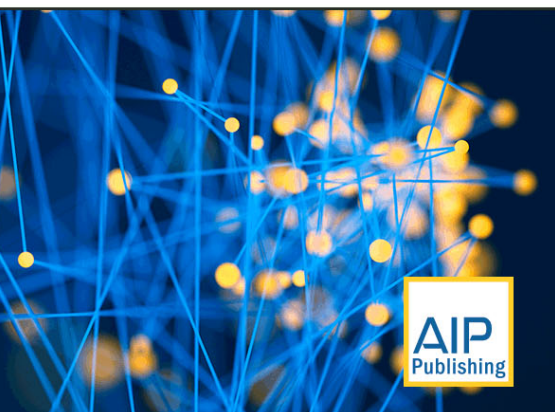




\title{
Thermodynamics of quasi-2D electron gas at BFO/Si interface probed with THz time-domain spectroscopy
}

\author{
Xiankuan Liu, ${ }^{1}$ Jiadong Zhang, ${ }^{1}$ Zeyu Zhang, ${ }^{1}$ Xian Lin, ${ }^{1}$ Yang Yu, ${ }^{1}$ Xiao Xing, ${ }^{1}$ \\ Zuanming Jin, ${ }^{1, \text { a) }}$ Zhenxiang Cheng, ${ }^{2}$ and Guohong $\mathrm{Ma}^{1, \mathrm{a})}$ \\ ${ }^{1}$ Department of Physics, Shanghai University, 99 Shangda Road, Shanghai 200444, People's Republic of China \\ ${ }^{2}$ Institute for Superconducting and Electronic Materials, University of Wollongong, New South Wales 2500, \\ Australia
}

(Received 11 June 2017; accepted 25 September 2017; published online 12 October 2017)

\begin{abstract}
An interface is constructed based on a bismuth ferrite oxide (BFO) thin film and p-type silicon, and the temperature dependence of the interface properties has been studied systematically using terahertz time-domain spectroscopy. The BFO/Si interface exhibits quasi-two-dimension electron gas (2DEG) transport in the temperature range of 80 to $140 \mathrm{~K}$ : the electrons at the interface possess large electron mobility $\left(\sim 10^{6} \mathrm{~cm}^{2} / \mathrm{V} \mathrm{s}\right)$ and long scattering time $(\sim 100 \mathrm{ps})$. As the temperature is higher than $140 \mathrm{~K}$, an abrupt decrease in THz interface conductivity is observed due to the breakdown of the 2DEG induced by the surface phase transition in the BFO thin film. Our result reveals that the interface formed between BFO and Si provides a special platform for designing and fabricating THz photonic devices. Published by AIP Publishing. https://doi.org/10.1063/1.4989667
\end{abstract}

Since the findings of two-dimensional electron gas (2DEG) in $\mathrm{LaAlO}_{3} / \mathrm{SrTiO}_{3}(\mathrm{LAO} / \mathrm{STO})$ heterostructures in $2004,{ }^{1}$ interface electronics based on perovskite oxide have attracted much attention in recent years due to the novel electronic, magnetic, ${ }^{2-8}$ and superconducting ${ }^{9,10}$ phenomena appearing at the interface of double layer perovskite oxide. It is also well known that $2 \mathrm{DEG}$ can be formed in semiconductor heterojunctions, for instance, GaAs/AlGaAs has tremendous applications in modern micro-electronics and photonics. The 2DEG formed at the interface of double-layer perovskite oxide is fundamentally different from that formed in the semiconductor heterojunction. If a semiconductor and a perovskite are brought together, a special interface might be formed, and the interface may have some interesting properties that the intrinsic materials do not have. For instance, the interface at LAO/STO shows conducting 2D electron gas, ${ }^{1}$ superconducting properties, ${ }^{9}$ and the coexistence of superconductivity and ferromagnetism $;{ }^{5}$ The interface at graphene $/ \mathrm{Si}^{11}$ and $\mathrm{BiFeO}_{3}(\mathrm{BFO}) / \mathrm{Si}^{12}$ dominates diode-like $\mathrm{THz}$ transmission at room temperature. As far as we know, there is little report on the interface properties of a perovskite grown on a semiconductor. ${ }^{12}$

Multiferroic $\mathrm{BiFeO}_{3}(\mathrm{BFO})$ possesses a perovskite structure and exhibits abundance of electronic and magnetic properties with temperature. Specifically, BFO has some attractive features such as large spontaneous polarization in both phases of a single crystal ${ }^{13,14}$ and a thin film. ${ }^{15,16}$ Phase transitions occurring at 140 and $200 \mathrm{~K}$ in the $\mathrm{BFO}$ thin film were observed ten years ago. ${ }^{17-19}$ The phase transition initially was attributed to the spin reorientation phase transition, i.e., the magnetization of iron ions switches from the $c$-axis to the $a$-axis with the decreasing temperature, which is similar to that in rare-earth orthoferrite. ${ }^{20,21}$ Later on, the origin of low temperature phase transition was confirmed as a

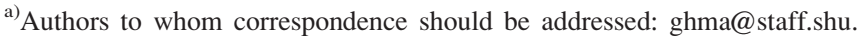
edu.cn and physics_jzm@shu.edu.cn
}

surface phase transition. ${ }^{21,22}$ These suggest that BFO not only has ferromagnetic and ferroelectric properties at room temperature but also may have interesting interface effects between the BFO thin film and other materials at low temperature. In the present study, by depositing the BFO film on a p-type silicon substrate, a heterostructure is constructed based on the BFO/Si interface. The temperature dependent electronic transport properties of the interface are investigated using $\mathrm{THz}$ time domain spectroscopy. Our experimental results demonstrated that upon increasing the temperature from 40 to $140 \mathrm{~K}$, the $\mathrm{BFO} / \mathrm{Si}$ interface shows a sharp increase in carrier mobility, carrier density, and scattering time. Moreover, BFO surface phase transition behavior is also confirmed to occur at around $140 \mathrm{~K}$ by monitoring the temperature dependence of $\mathrm{THz}$ interface conductivity. Our study reveals that $2 \mathrm{D}$ electron gas can be confined at the perovskite/semiconductor interface at proper temperature, which not only can be used to design electronic and photonic devices but also provides a platform to test the fundamental properties of the perovskite itself.

Particularly, $\mathrm{La}$ and $\mathrm{Nb}$ codoped $\mathrm{BiFeO}_{3}$ (namely $\mathrm{Bi}_{0.8} \mathrm{La}_{0.2} \mathrm{Fe}_{0.99} \mathrm{Nb}_{0.01} \mathrm{O}_{3}$, for simplicity, we use "BFO" throughout the text) films were deposited on p-type (001) silicon and (001) $\mathrm{MgO}$ substrates by the pulsed laser deposition method, and the $\mathrm{BFO} / \mathrm{MgO}$ hybrid structure was fabricated as a reference in contrast to its $\mathrm{BFO} / \mathrm{Si}$ counterpart. It is worth mentioning that the $\mathrm{La}$ and $\mathrm{Nb}$ codoped $\mathrm{BFO}$ film shows a much better electrical polarization than the intrinsic $\mathrm{BiFeO}_{3}$ film by decreasing the leakage current of ferroelectrics. ${ }^{23}$ During the deposition of $\mathrm{BFO}$, the substrates of $\mathrm{Si}$ and $\mathrm{MgO}$ remained at $550{ }^{\circ} \mathrm{C}$ and then cooled down to room temperature following rapid thermal processing. During the deposition, the dynamic oxygen flow pressure was kept at 20 mTorr. The details of the sample fabrication have been reported elsewhere. ${ }^{23}$ The thickness of the BFO film is about $200 \pm 15 \mathrm{~nm}$, and the $0.4 \mathrm{~mm}$-thick silicon substrate was lightly doped by boron to form a p-type semiconductor ${ }^{24}$ with a dopant 
concentration of $\sim 10^{14} \mathrm{~cm}^{-3}$. 2 The thermodynamic behaviors of the $\mathrm{BFO} / \mathrm{Si}$ interface were investigated by $\mathrm{THz}$ time domain spectroscopy in the transmission configuration. The details of the setup are presented in supplementary material 1.

Figure 1 shows the $\mathrm{THz}$ electric field transmission in vacuum (as a reference) and the $\mathrm{BFO} / \mathrm{Si}$ heterostructure with four selected temperatures. Apart from the main pulse localized around $\sim 15 \mathrm{ps}$, a weak signal appears around $24 \mathrm{ps,}$ which corresponds to the second reflection (echo pulse) of the main pulse at each temperature. Upon increasing the temperature from 40 to $140 \mathrm{~K}$, the amplitude of the main pulse is seen to decrease, which suggests that the conductivity of the sample increases with temperature. In contrast to the amplitude transmission, the $\mathrm{THz}$ phase shows a negative phase shift by increasing the temperature between 40 and $140 \mathrm{~K}$, which suggests that the transmitted amplitude and the phase shift may come from different contributions. Compared to the main pulse, the "echo pulse" exhibits a zero-phase-shift at a low temperature of $40 \mathrm{~K}$, and it undergoes a $\pi$ phase shift for the cases of $100 \mathrm{~K}$ and $140 \mathrm{~K}$, while, at a high temperature of $300 \mathrm{~K}$, the "echo pulse" goes back to the zero-phaseshift again with the main pulse. Supplementary material 3 presents more details about the phase shifts of the "echo pulse" as a function of temperature in the hybrid structure. We have also checked the amplitude transmission and the phase shift of $\mathrm{THz}$ signals through a $0.4 \mathrm{~mm}-\mathrm{Si}$ wafer (ptype) and a BFO thin film on the $0.5 \mathrm{~mm}-\mathrm{MgO}$ substrate at various temperatures (supplementary material 4). Both the $\mathrm{Si}$ wafer and the $\mathrm{BFO} / \mathrm{MgO}$ heterostructure do not exhibit temperature dependent behavior at the investigated $\mathrm{THz}$ frequency. The strong temperature dependence of $\mathrm{THz}$ amplitude transmission in the $\mathrm{BFO} / \mathrm{Si}$ heterostructure suggests that the conductivity change comes from the BFO-Si interface effect, and the conductivity change in individual BFO films and $\mathrm{Si}$ substrates with temperature is negligible. In particular, the $\pi$ phase shift of echo pulses occurring at 100 and $140 \mathrm{~K}$ in the hybrid structure indicates that the $\mathrm{BFO} / \mathrm{Si}$ interface has high conductivity between these two temperatures. Figure 1(b) shows the Fourier transforms of Fig. 1(a) in the time range of 0 to $22 \mathrm{ps}$; the $\mathrm{BFO} / \mathrm{Si}$ heterostructure exhibits a broadband frequency modulation with temperature.

Figures 1(c) and 1(d) show the refractive index $(n)$ and absorption $(\alpha)$ of $\mathrm{BFO} / \mathrm{Si}$ in the temperature range of 40 to $140 \mathrm{~K}$ ( $n$ and $\alpha$ in the temperature range of $150-300 \mathrm{~K}$ are presented in supplementary material 2 and 3 . The inset of Fig. 1(c) shows the temperature dependence of $n$ at three selected frequencies of $0.4,0.7$, and $1.0 \mathrm{THz}$. A nonmonotonous temperature dependence of $n$ is observed, and the minimum $n$ occurs in the temperature range between $110 \mathrm{~K}$ and $140 \mathrm{~K}$ depending on the detecting frequency. The inset of Fig. 1(d) presents the temperature dependence of $\alpha$ at $0.4,0.7$, and $1.0 \mathrm{THz}$. We observe absorption peaks at around $140 \mathrm{~K}$ for all the selected frequencies. The opposite temperature dependent trend between $n$ and $\alpha$ gives a solid evidence that the two parameters arise from different mechanisms in BFO/Si. The interface effect due to the formation of 2DEG dominates the absorption, while carrier transportation between the $\mathrm{BFO}$ film and the $\mathrm{BFO} / \mathrm{Si}$ interface plays an important role in the temperature dependent refractive index. Thus, it is reasonable to ignore the phase term during the calculation of the temperature dependent conductivity of the 2DEG.

In order to understand the thermodynamical formation of the quasi-2DEG at the interface of $\mathrm{BFO} / \mathrm{Si}$, we present the schematic diagram of the energy bandgap for isolated $\mathrm{BFO}$ and $\mathrm{Si}$, an ideal $\mathrm{BFO} / \mathrm{p}$-type $\mathrm{Si}$ heterojunction at thermal equilibrium, and the heterojunctions of BFO/Si at $40 \mathrm{~K}$ and $100 \mathrm{~K}$, respectively (supplementary material 5). The energy bandgap alignment for the $\mathrm{BFO} / \mathrm{Si}$ heterostructure is used to illustrate the formation of the $2 \mathrm{DEG}$ at the interface. The energy bandgap diagram presented in Fig. S3 (supplementary material, part 3) suggests that the 2DEG can be formed between the BFO and the Si substrate with varying temperatures. $\mathrm{THz}$ spectroscopy spectra presented in Fig. 1 can be
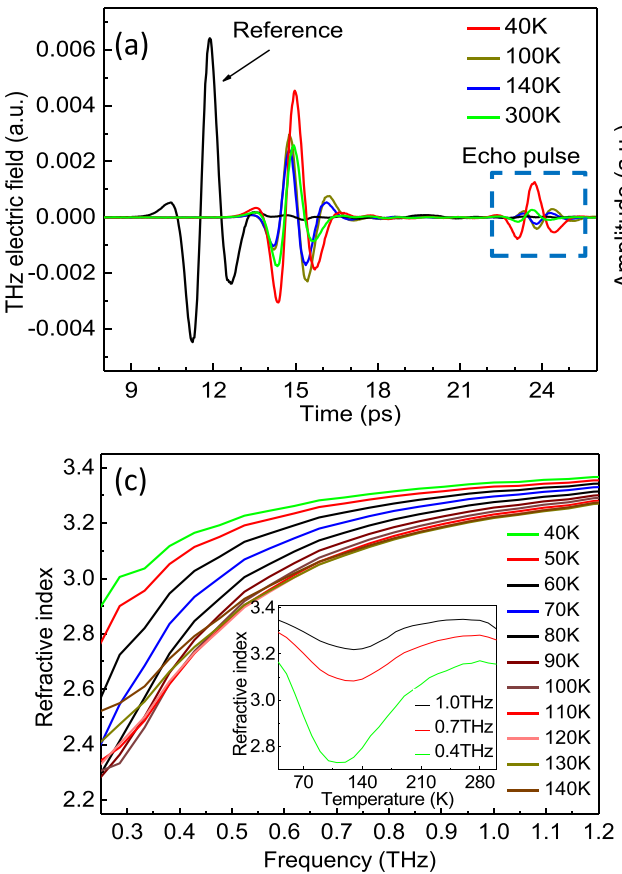
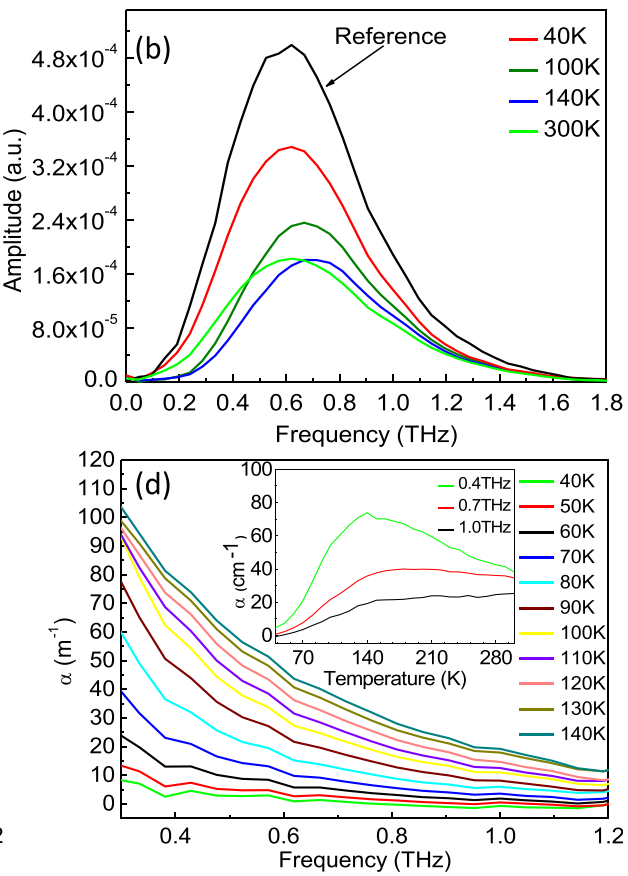

FIG. 1. (a) Transmitted THz electric field of the reference (vacuum) and $\mathrm{BFO} / \mathrm{Si}$ at selected temperatures of 40 , 100,140 , and $300 \mathrm{~K}$. (b) Fourier transform of (a) in the time range of 0 to 22 ps. (c) Refractive index of $\mathrm{BFO} / \mathrm{Si}$ in the temperature range of 40 to $140 \mathrm{~K}$; the inset shows the refractive index as a function of temperature at three selected frequencies. (d) Absorption coefficient of $\mathrm{BFO} / \mathrm{Si}$ in the temperature range of 40 to $140 \mathrm{~K}$; the inset shows the absorption coefficient vs temperature at the three selected frequencies. 
well interpreted with the temperature dependence of electron transport of the 2DEG. In order to elaborate the 2D electron character further at the $\mathrm{BFO} / \mathrm{Si}$ interface, electron conductivity, mobility, and scattering time of the $2 \mathrm{DEG}$ in $\mathrm{BFO} / \mathrm{Si}$ are analyzed by $\mathrm{THz}$ spectroscopy in the following part. In fact, the anomaly temperature dependence of the refractive index in the $\mathrm{BFO} / \mathrm{Si}$ hybrid structure is derived from the electron transportation between the $\mathrm{BFO}$ film and the $\mathrm{BFO} / \mathrm{Si}$ interface rather than from the phase shift of the 2DEG. In addition, considering that the $2 \mathrm{DEG}$ shows a very large absorption coefficient and very narrow interface space (a few $\mathrm{nm}$ ), the phase shift of 2DEG can be neglected during the calculation of the conductivity. By taking $\mathrm{THz}$ transient at $40 \mathrm{~K}$ as a reference, the temperature dependence of conductivity changes of the $2 \mathrm{DEG}$ is calculated by ${ }^{25}$

$$
\Delta \sigma_{\text {sheet }}(\omega)=\frac{2}{Z_{0}} \times\left[\frac{1}{t(T, \omega)}-1\right],
$$

where $Z_{0}$ is the impedance of free space. $t(T, \omega)=\frac{A(T, \omega)}{A(40, \omega)}$ is the $\mathrm{THz}$ amplitude ratio between $\mathrm{A}(\mathrm{T}, \omega)$ at temperature $T$ and $\mathrm{A}(40, \omega)$ at $40 \mathrm{~K}$. Figures $2(\mathrm{a})$ and $2(\mathrm{~b})$ show the sheet conductivity change of the 2DEG in the temperature range of 50 to $300 \mathrm{~K}$. For clarity, Fig. 2(a) shows the low temperature range of 50 to $140 \mathrm{~K}$ and Fig. 2(b) shows the high temperature range of 150 to $300 \mathrm{~K}$ (supplementary material 6). It is seen that the dispersion of sheet conductivity changes with temperature shows a different trend in low and high temperature ranges. The solid lines in Figs. 2(a) and 2(b) are the fitting curves with the Drude-Smith model, as given by Eq. (2),

$$
\operatorname{Re}\left[\tilde{\sigma}(\omega)=\frac{\varepsilon_{0} \omega_{p}^{2} \tau}{1-i \omega \tau}\left(1+\frac{c_{0}}{1-i \omega \tau}\right)\right] .
$$

Here, $\varepsilon_{0}, \omega_{p}$, and $\tau$ are the permittivity in vacuum, plasma frequency, and carrier scattering time, respectively. $\mathrm{c}_{0}$ is the backscattering factor (between 0 and -1 ). $\mathrm{c}_{0}=0$ means that all carriers are free, and Eq. (2) is reduced to a Drude model; $\mathrm{c}_{0}=-1$ means that all carriers are localized. The fitting parameter $\mathrm{c}_{0}$ as a function of temperature is shown in Fig. 2(c). As the temperature is below $140 \mathrm{~K}, \mathrm{c}_{0}=0 ; \mathrm{c}_{0}$ approaches -1 when the temperature is higher than $200 \mathrm{~K}$. From 140 to $200 \mathrm{~K}, \mathrm{c}_{0}$ decreases from 0 to -1 as temperature increases. From the temperature dependence of $\mathrm{c}_{0}$, we find that the carriers at the $\mathrm{BFO} / \mathrm{Si}$ interface are free in 2-dimension when the temperature is below $140 \mathrm{~K}$, carriers undergo localization with the increasing temperature, and all carriers are localized when the temperature is higher than $200 \mathrm{~K}$. Figure 2(d) shows the temperature dependence of fitting parameters of plasma frequency $\omega_{\mathrm{p}}$ and scattering time $\tau$. Both parameters show a nonlinear temperature dependence. In the low temperature range below $80 \mathrm{~K}$, the magnitudes of $\omega_{\mathrm{p}}$ and $\tau$ increase with temperature. This process corresponds to the formation process of the 2DEG with temperature. As a matter of fact, the carrier density in the potential well is proportional to $n_{0} \exp \left(-\mathrm{e} \gamma_{\mathrm{b}} / \mathrm{K}_{\mathrm{B}} \mathrm{T}\right)$, with $\mathrm{n}_{0}, \gamma_{\mathrm{b}}, \mathrm{K}_{\mathrm{B}}$, and $\mathrm{T}$ being the carrier density in the BFO thin film, barrier voltage [as shown in Fig. 3(b)], Boltzmann constant, and temperature, respectively. As $\mathrm{T}>80 \mathrm{~K}$, most of the carriers can overcome the barrier and enter into the potential well and the 2DEG is formed; as a result, the conductivity, plasma frequency, and scattering time show a sharp increase as shown in Fig. 2(d). In the temperature range between 80 and $140 \mathrm{~K}$, both $\omega$ and $\tau$ increase slightly with temperature, indicating that the $2 \mathrm{DEG}$ is stable in this temperature range. Upon increasing the temperature further, both magnitudes of $\omega$ and $\tau$ show a sharp decrease at around $140 \mathrm{~K}$. When $\mathrm{T}>140 \mathrm{~K}, \omega$ and $\tau$ decrease slightly with temperature, and the fitting parameters $c_{0}$ decrease from zero to negative, which demonstrates that carrier localization occurs at high temperature.

The fitting parameters, $\omega_{\mathrm{p}}$ and $\tau$, are also utilized to calculate the carrier mobility $(\Delta \mu)$ and $d c$ conductivity $\left(\Delta \sigma_{\mathrm{dc}}\right)$ changes of the 2DEG at the BFO/Si interface by $\mu=\frac{e \tau}{m *}$ and $\sigma_{\mathrm{dc}}=\varepsilon_{0} \omega_{p}^{2} \tau$, with $\mathrm{m} *$ and $\varepsilon_{0}$ being the effective mass of electrons at the $\mathrm{BFO} / \mathrm{Si}$ interface and the dielectric constant of vacuum, respectively. As shown in Figs. 3(a) and 3(b), upon
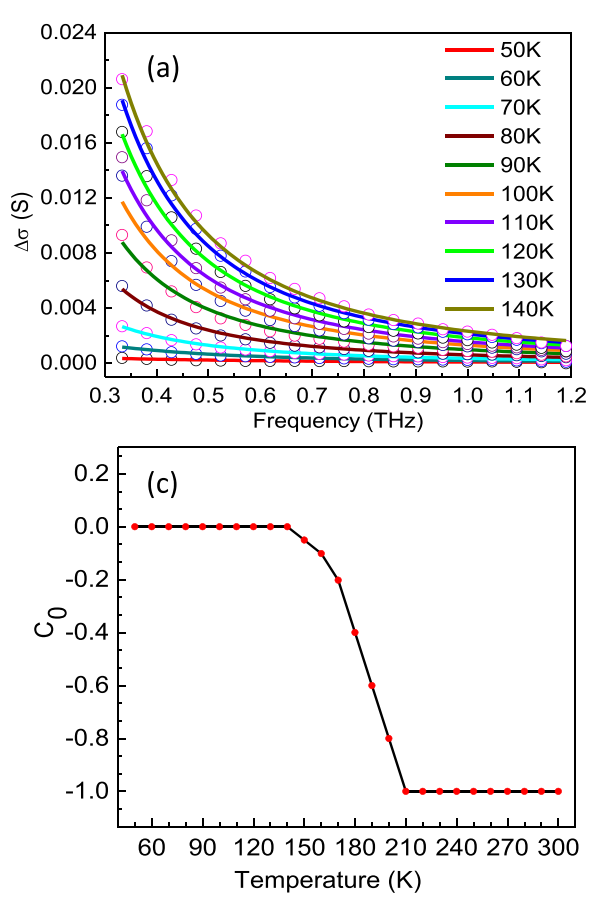
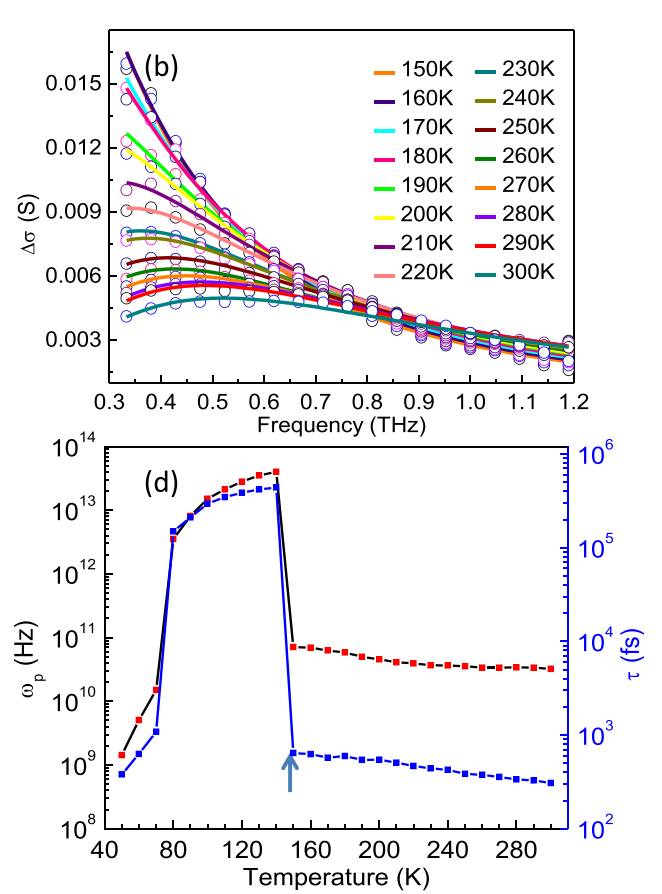

FIG. 2. Interface-sheet conductivity change (color circles) of the $\mathrm{BFO} / \mathrm{Si}$ (a) from 50 to $140 \mathrm{~K}$ and (b) from 150 to $300 \mathrm{~K}$. The solid lines are the fitting curves by Eq. (2). (c) The fitting parameters $\mathrm{c}_{0}$ are plotted as a function of temperature. (d) The plasma frequency $\omega_{\mathrm{p}}$ and scattering time $\tau$ are plotted as a function of temperature. 


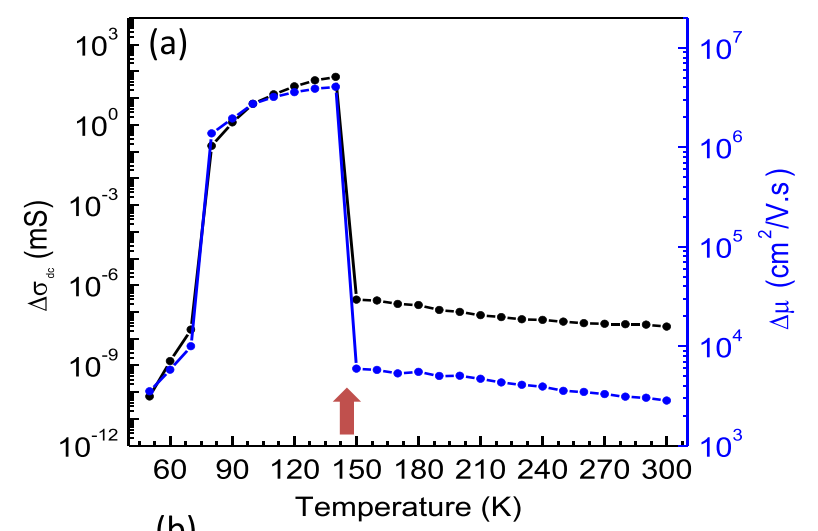

(b)

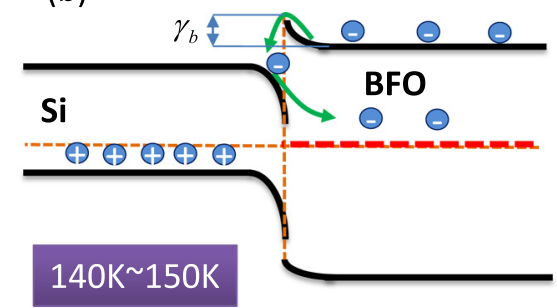

FIG. 3. (a) Electronic $d c$ conductivity and mobility changes in BFO/Si from 50 to $300 \mathrm{~K}$. (b) Diagram of the electron tranport process between the BFO film and the BFO/Si interface. The red dashed line in the diagram represents the defect energy level, which crosses over with the Fermi energy at $140 \mathrm{~K}$ and above.

the increasing temperature from $50 \mathrm{~K}$ to $80 \mathrm{~K}$, a sharp increase in carrier mobility is observed, which reveals the $2 \mathrm{DEG}$ formation process at the $\mathrm{BFO} / \mathrm{Si}$ interface. As $\mathrm{T}>140 \mathrm{~K}$, more than 3 orders of magnitude of carrier mobility decrease is observed. $\sigma_{\mathrm{dc}}$ shows a similar temperature dependence to $\mu$. Within the temperature range of $80-140 \mathrm{~K}$, the carriers in the heterostructure show large mobility, high $a c$ and $d c$ conductivity, and long scattering time, and these unique properties of carriers are the strong evidence of the carriers confined in the $2 \mathrm{D}$ state.

The carrier transport properties show a sharp decrease as $\mathrm{T}>140 \mathrm{~K}$, as indicated by arrows in Figs. 2(d) and 3(a), which demonstrate the breakdown of the 2DEG. It is noted that the BFO film undergoes phase transition at $\sim 140 \mathrm{~K}$. It is reasonable to infer that the sharp change in conductivity near $140 \mathrm{~K}$ may have close correlation with the phase transition in the BFO thin film. Cazayous et al. first reported the phase transition at $140 \mathrm{~K}$, which was assigned to a spin reorientation phase transition in the BFO thin film. Recently, Jarrier et al. re-investigated the phase transition of BFO in a wide range of temperatures, and they assigned the transition occurring at $140 \mathrm{~K}$ as a surface phase transition. ${ }^{22}$ Two main features have been identified for the occurrence of surface phase transition in BFO: (i) a sharp volume change without the actual change in symmetry and (ii) sharp emission of charge (pyroelectric-like current) and maximum in the conductivity of the surface, which are consistent with a crossover between an impurity level and the Fermi level and structural and magnetic disorder at $140 \mathrm{~K}^{22}$ As the BFO thin film undergoes the phase transition near $140 \mathrm{~K}$, the Fermi level at the BFO interface may experience an abrupt change and the interfacial defect state below the Fermi level might cross over above it. As shown in Fig. 3(b), the charge is thus released in the defect state of BFO, and the surface charge density in the defect state can be increased greatly above $140 \mathrm{~K}$. As a result, electrons cannot be restricted any more in the potential well formed at the $\mathrm{BFO} / \mathrm{Si}$ interface. Thus, the $\mathrm{THz}$ conductivity shows an abrupt decrease at $\mathrm{T}=140 \mathrm{~K}$. A first-principles density-functional calculation was performed by determining defects existing on the surface, and the origin of the defects most likely comes from the Bi vacancies. ${ }^{22}$

In summary, by employing $\mathrm{THz}$ spectroscopy, we have investigated the temperature dependence of the interface effect of the BFO/Si heterostructure. The heterostructure has a high carrier mobility $\left(>10^{6} \mathrm{~cm}^{2} / \mathrm{V} \mathrm{s}\right)$ and long scattering time ( $>100 \mathrm{ps}$ ) within the temperature range of $80-140 \mathrm{~K}$, suggesting a stable $2 \mathrm{DEG}$ phase. The conductivity decreases sharply at $\mathrm{T}>140 \mathrm{~K}$, which indicates the breakdown of the 2DEG due to the combined effects of 2DEG and surface phase transition in the BFO film. Our results demonstrate that the formation mechanism of $2 \mathrm{DEG}$ at the interface of $\mathrm{BFO} / \mathrm{Si}$ is similar to that in the conventional semiconductor heterojunction. Our findings could provide insights into the understanding of the 2DEG in the double layer perovskite heterostructure and also pave the way toward potential multiferroic/semiconductor hybrid devices with multiple functionalities.

See supplementary material for complete temperature dependent $\mathrm{THz}$ spectroscopies of the samples.

This work was supported by the National Natural Science Foundation of China (Nos. 11674213, 11604202, and 61735010), the Young Eastern Scholar (No. QD2015020), the "Chen Guang" project (No. 16CG45), and the Universities Young Teachers Training Funding Program (No. ZZSD15098).

${ }^{1}$ A. Ohtomo and H. Y. Hwang, Nature 427(6973), 423 (2004).

${ }^{2}$ A. X. Wang, G. Baskaran, Z. Q. Liu, J. Huijben, J. B. Yi, A. Annadi, A. Roy Barman, A. Rusydi, S. Dhar, Y. P. Feng, J. Ding, H. Hilgenkamp, and T. Venkatesan, Nat. Commun. 2, 188 (2011).

${ }^{3}$ J. A. Bert, B. Kalisky, C. Bell, M. Kim, Y. Hikita, H. Y. Hwang, and K. A. Moler, Nat. Phys. 7(10), 767 (2011).

${ }^{4}$ L. Li, C. Richter, J. Mannhart, and R. C. Ashoori, Nat. Phys. 7(10), 762 (2011).

${ }^{5}$ D. A. Dikin, M. Mehta, C. W. Bark, C. M. Folkman, C. B. Eom, and V. Chandrasekhar, Phys. Rev. Lett. 107(5), 056802 (2011).

${ }^{6}$ M. Gabay and J.-M. Triscone, Nat. Phys. 9(10), 610 (2013).

${ }^{7}$ L. Weston, X. Y. Cui, S. P. Ringer, and C. Stampfl, Phys. Rev. Lett. 113(18), 186401 (2014).

${ }^{8}$ D. Doennig and R. Pentcheva, Sci. Rep. 5, 7909 (2015).

${ }^{9}$ N. Reyren, S. Thiel, A. D. Caviglia, L. Fitting Kourkoutis, G. Hammerl, C. Richter, C. W. Schneider, T. Kopp, A.-S. Rüetschi, and D. Jaccard, Science 317(5842), 1196 (2007).

${ }^{10}$ Y.-L. Han, S.-C. Shen, J. You, H.-O. Li, Z.-Z. Luo, C.-J. Li, G.-L. Qu, C.-M. Xiong, R.-F. Dou, and L. He, Appl. Phys. Lett. 105(19), 192603 (2014).

${ }^{11}$ Q. Li, Z. Tian, X. Zhang, R. Singh, L. Du, J. Gu, J. Han, and W. Zhang, Nat. Commun. 6, 7082 (2015).

${ }^{12}$ X. Liu, Z. Zhang, X. Lin, K. Zhang, Z. Jin, Z. Cheng, and G. Ma, Opt. Express 24(23), 26618 (2016).

${ }^{13}$ D. Lebeugle, D. Colson, A. Forget, M. Viret, P. Bonville, J.-F. Marucco, and S. Fusil, Phys. Rev. B 76(2), 024116 (2007).

${ }^{14}$ D. Lebeugle, D. Colson, A. Forget, and M. Viret, Appl. Phys. Lett. 91(2), 022907 (2007).

${ }^{15}$ J. B. N. J. Wang, J. B. Neaton, H. Zheng, V. Nagarajan, S. B. Ogale, B. Liu, D. Viehland, V. Vaithyanathan, D. G. Schlom, and U. V. Waghmare, Science 299(5613), 1719 (2003).

${ }^{16}$ W. Eerenstein, F. D. Morrison, J. Dho, M. G. Blamire, J. F. Scott, and N. D. Mathur, Science 307(5713), 1203 (2005).

${ }^{17}$ M. K. Singh, W. Prellier, M. P. Singh, R. S. Katiyar, and J. F. Scott, Phys. Rev. B 77(14), 144403 (2008). 
${ }^{18}$ M. Cazayous, Y. Gallais, A. Sacuto, R. De Sousa, D. Lebeugle, and D. Colson, Phys. Rev. Lett. 101(3), 037601 (2008).

${ }^{19}$ M. K. Singh, R. S. Katiyar, and J. F. Scott, J. Phys.: Condens. Matter 20(25), 252203 (2008).

${ }^{20}$ S. Venugopalan, M. Dutta, A. K. Ramdas, and J. P. Remeika, Phys. Rev. B 31(3), 1490 (1985).

${ }^{21}$ X. Martí, P. Ferrer, J. Herrero-Albillos, J. Narvaez, V. Holy, N. Barrett, M. Alexe, and G. Catalan, Phys. Rev. Lett. 106(23), 236101 (2011).
${ }^{22}$ R. Jarrier, X. Marti, J. Herrero-Albillos, P. Ferrer, R. Haumont, P. Gemeiner, G. Geneste, P. Berthet, T. Schülli, and P. Cevc, Phys. Rev. B 85(18), 184104 (2012).

${ }^{23}$ Z. Cheng, X. Wang, S. Dou, H. Kimura, and K. Ozawa, Phys. Rev. B 77(9), 092101 (2008).

${ }^{24}$ Z. Jin, Y. Xu, Z. Zhang, X. Lin, G. Ma, Z. Cheng, and X. Wang, Appl. Phys. Lett. 101(24), 242902 (2012).

${ }^{25}$ J. Lloyd-Hughes and T.-I. Jeon, J. Infrared, Millimeter, Terahertz Waves 33(9), 871 (2012). 